\title{
VARIATIONAL B-SPLINE LEVEL-SET METHOD FOR FAST IMAGE SEGMENTATION
}

\author{
O. Bernard, D. Friboulet \\ CREATIS, CNRS UMR 5220, Inserm U630, \\ National Institute of Applied Sciences \\ 7, avenue Jean Capelle \\ 69621 Villeurbanne Cedex, France
}

\author{
P. Thevenaz, M. Unser \\ Biomedical Imaging Group \\ Swiss Federal Institute of \\ Technology Lausanne (EPFL) \\ CH-1015 Lausanne-EPFL, Switzerland
}

\begin{abstract}
In the field of image segmentation, most of level-set-based active contour approaches are based on a discrete representation of the associated implicit function. We present in this paper a different formulation where the level-set is modelled as a continuous parametric function expressed on a B-spline basis. Starting from the Mumford-Shah energy functional, we show that this formulation allows computing the solution as a restriction of the variational problem on the space spanned by the B-splines. As a consequence, the minimization of the functional is directly obtained in terms of the B-splines parameters. We also show that each step of this minimization may be expressed through a convolution operation. Because the B-spline functions are separable, this convolution may in turn be performed as a sequence of simple $1 \mathrm{D}$ convolutions, which yields a very efficient algorithm. The behaviour of this approach is illustrated on biomedical images from various fields.
\end{abstract}

Index Terms - Level-set, B-spline, Variational methods

\section{INTRODUCTION}

Level-set based formulations have become a well-established tool in the field of image processing $[1,2]$. In image segmentation, level-set-based methods corresponds to a class of deformable models where the shape to be recovered is captured by propagating an interface represented by the zero levelset of a smooth function (usually called the level-set function). The evolution at the interface is generally derived from a variational formulation: the segmentation problem is expressed as the minimization of an energy functional reflecting the properties of the objects to be recovered. Formally, the minimization of this functional provides the evolution of the level-set function as a time dependent partial differential equation (PDE), which is usually solved using finite differences schemes [2].

In contrast with this now well-known discrete scheme, we present a continuous approach, where the level-set function is expressed as a continuous parametric function using Bsplines. A similar idea has recently been developed in [3, 4], where the continuous representation is based on radial basis functions (RBFs), which are then used to solve the level-set PDE. In contrast with these approaches, we start from the initial level-set energy functional and propose to specify the solution as a restriction of the variational problem on a space spanned by B-spline basis functions. As a consequence, the minimization of the functional is directly obtained in term of the B-splines parameters.

The paper is structured as follows: In Section 2, we recall the general form of the level-set energy functional, describe the B-spline formulation of the problem and derive the minimization of the functional in terms of the B-spline parameters. In particular we show how the gradient of the functional, hence the evolution of the level-set, may be expressed as a convolution. We discuss implementation issues of the method in Section 3. We show in particular how reinitialization of the level-set may be avoided by normalizing the B-splines parameters. In Section 4, we evaluate the behavior of the method using biomedical images. The main conclusions and perspectives of this work are given in Section 5 .

\section{PROBLEM FORMULATION}

Let $\Omega$ be a bounded open subset of $\mathbb{R}^{d}$ and let $f: \Omega \mapsto \mathbb{R}$ be a given $d$-dimensional image. In the level-set formalism, the evolving interface $\Gamma \subset \mathbb{R}^{d}$ is represented as the zero level-set of a Lipschitz-continuous function $\phi$ that satisfies

$$
\begin{cases}\phi(\mathbf{x})>0, & \forall \mathbf{x} \in \Omega_{\text {in }} \\ \phi(\mathbf{x})<0, & \forall \mathbf{x} \in \Omega_{\text {out }} \\ \phi(\mathbf{x})=0, & \forall \mathbf{x} \in \Gamma,\end{cases}
$$

where $\Omega_{\text {in }}$ is a region in $\Omega$ bounded by $\Gamma=\partial \Omega_{\text {in }}$. The region $\Omega_{\text {out }}$ is defined as $\Omega \backslash \Omega_{\text {in }}$.

\subsection{Energy criterion}

We consider in the following the classical problem of segmenting one object (possibly having several nonconnected components) from the background. This problem is typically handled by the evolution of one level-set whose steady state 
partitions the image into two regions delimiting the boundaries of the object to be segmented. In this framework, we use the well-known Chan-Vese functional [5], which aims at partitioning the image into regions with piecewise constant intensity. This approach corresponds to a particular case of Mumford-Shah functional [6], known as the minimal partitioning problem. The corresponding functional is given as:

$$
\begin{aligned}
& J\left(\phi, \mu_{1}, \mu_{2}\right)=\int_{\Omega}\left(f(\mathbf{x})-\mu_{1}\right)^{2} H_{\epsilon}(\phi(\mathbf{x})) \mathrm{d} x_{1} \cdots \mathrm{d} x_{d} \\
& +\int_{\Omega}\left(f(\mathbf{x})-\mu_{2}\right)^{2}\left(1-H_{\epsilon}(\phi(\mathbf{x}))\right) \mathrm{d} x_{1} \cdots \mathrm{d} x_{d} \\
& +\nu \int_{\Omega}\|\boldsymbol{\nabla} \phi(\mathbf{x})\| \delta_{\epsilon}(\phi(\mathbf{x})) \mathrm{d} x_{1} \cdots \mathrm{d} x_{d}
\end{aligned}
$$

where $\left(\mu_{1}, \mu_{2}\right)$ are the two parameters of the energy function, and where $\nu$ is a hyper-parameter that balances the influence of the regions terms (first and second integrals) and of the contour term (third integral). The functions $H_{\epsilon}(\cdot)$ and $\delta_{\epsilon}(\cdot)$ are $C^{\infty}$ regularized versions of the Heaviside and of the Dirac functions, the latter being the derivative of the former.

\subsection{B-spline level-set model}

The model is obtained by expressing the level-set function $\phi(\mathbf{x})$ as the linear combination of B-spline basis functions [7]

$$
\phi(\mathbf{x})=\sum_{\mathbf{k} \in \mathbb{Z}^{d}} c[\mathbf{k}] \beta^{n}\left(\frac{\mathbf{x}}{h}-\mathbf{k}\right) .
$$

Here, $\beta^{n}(\cdot)$ is the uniform symmetric $d$-dimensional B-spline of degree $n$. This function is separable and is built as the product of $d$ one-dimensional B-splines, so that $\beta^{n}(\mathbf{x})=$ $\prod_{j=1}^{d} \beta^{n}\left(x_{j}\right)$. The knots of the B-splines are located on a grid spanning $\Omega$, with a regular spacing. The coefficients of the B-spline representation are gathered in $c[\mathbf{k}]$.

\subsection{Functional minimization}

In order to reach a local minimum of energy criterion (4), we use a conventional Expectation Maximization $(E M)$ technique. First, keeping $\phi$ (i.e. $c[\mathbf{k}]$ ) fixed, we minimize the energy criterion with respect to $\mu_{1}$ and $\mu_{2}$. These parameters are then given as:

$$
\left\{\begin{aligned}
\mu_{1} & =\frac{\int_{\Omega} f(\mathbf{x}) H_{\epsilon}(\phi(\mathbf{x})) \mathrm{d} x_{1} \cdots \mathrm{d} x_{d}}{\int_{\Omega} H_{\epsilon}(\phi(\mathbf{x})) \mathrm{d} x_{1} \cdots \mathrm{d} x_{d}} \\
\mu_{2} & =\frac{\int_{\Omega} f(\mathbf{x})\left(1-H_{\epsilon}(\phi(\mathbf{x}))\right) \mathrm{d} x_{1} \cdots \mathrm{d} x_{d}}{\int_{\Omega}\left(1-H_{\epsilon}(\phi(\mathbf{x}))\right) \mathrm{d} x_{1} \cdots \mathrm{d} x_{d}} .
\end{aligned}\right.
$$

Then, keeping $\mu_{1}$ and $\mu_{2}$ fixed, we minimize the energy criterion with respect to the level-set model. In the classical variational settings, this step is performed using either EulerLagrange equations [5] or the Fréchet/Gâteaux derivatives [8, 9]. In contrast with these approaches, we use the B-spline formulation (5) and perform the minimization with respect to the B-spline coefficients $c[\mathbf{k}]$. Such minimization implies computing the derivatives of (4) with respect to each B-spline coefficient $c\left[\mathbf{k}_{\mathbf{0}}\right]$. These derivatives may be expressed as:

$$
\frac{\partial J}{\partial c\left[\mathbf{k}_{\mathbf{0}}\right]}=\int_{\Omega} w(\mathbf{x}) \beta^{n}\left(\frac{\mathbf{x}}{h}-\mathbf{k}_{\mathbf{0}}\right) \mathrm{d} x_{1} \cdots \mathrm{d} x_{d},
$$

where

$$
\begin{gathered}
w(\mathbf{x})=\left(\left(f(\mathbf{x})-\mu_{1}\right)^{2}-\left(f(\mathbf{x})-\mu_{2}\right)^{2}-\right. \\
\left.\nu \operatorname{div}\left(\frac{\boldsymbol{\nabla} \phi(\mathbf{x})}{\|\boldsymbol{\nabla} \phi(\mathbf{x})\|}\right)\right) \delta_{\epsilon}(\phi(\mathbf{x})) .
\end{gathered}
$$

Here, $w(\mathbf{x})$ reflects the features of the object to be segmented and will be called the feature function in the sequel. The minimization of energy criterion (4) with respect to the B-spline coefficients does not lead to a closed-form solution. In order to obtain a local minimum, we then perform a gradient descent method which yields

$$
\mathbf{c}^{(i+1)}=\mathbf{c}^{(i)}-\lambda \nabla_{\mathrm{c}} J\left(\mathbf{c}^{(i)}\right),
$$

where $\nabla_{\mathrm{c}}$ corresponds to the gradient of the energy relative to the B-spline coefficients. The corresponding expression is given as:

$$
\nabla_{\mathrm{c}} J=\frac{\partial J}{\partial c[\mathbf{k}]}=\int_{\Omega} w(\mathbf{x}) \beta^{n}\left(\frac{\mathbf{x}}{h}-\mathbf{k}\right) \mathrm{d} x_{1} \cdots \mathrm{d} x_{d} .
$$

This last equation shows that the computation of the gradient of $J$ with respect to a set of B-spline coefficients may be interpreted as convolving the feature function $w(\mathbf{x})$ with the B-spline $\beta^{n}(\mathbf{x})$, and sampling the result on a grid spanning $\Omega$, with a regular spacing.

\subsection{Discretization: gradient calculation as a discrete sep- arable convolution}

In practice, the feature function is usually available only on a discrete grid. Let us call $w[\mathbf{u}]$ the corresponding discrete feature function, with $\mathbf{u} \in \mathbb{Z}^{d}$. By applying the discrete B-spline formulation of [10], we immediately obtain the discrete version of (10). The centered $d$-dimensional discrete B-spline of degree $n$ is noted $b^{n}[\mathbf{u}]$; it is obtained by sampling its continuous version $\beta^{n}(\mathbf{x})$ at integer values. Similar to its continuous counterpart, the sequence $b^{n}[\cdot]$ is separable and is built as the product of $d$ one-dimensional B-splines, so that $b^{n}[\mathbf{u}]=\prod_{j=1}^{d} b^{n}\left[u_{j}\right]$. The discrete version of the formulation is then obtained from (10) as

$$
\left\langle\nabla_{\mathrm{c}} J\right\rangle[\mathbf{k}]=\left\langle\frac{\partial J}{\partial c[\mathbf{k}]}\right\rangle=\sum_{\mathbf{u} \in \mathbb{Z}^{d}} w[\mathbf{u}] b_{h}^{n}(\mathbf{u}-h \mathbf{k}) .
$$

The energy gradient thus corresponds to the convolution of the feature image and the B-spline i.e.

$$
\left\langle\nabla_{\mathrm{c}} J\right\rangle[\mathbf{k}]=\left(w * b_{h}^{n}\right)[m \mathbf{k}] .
$$


This last expression provides an efficient way of calculating the gradient and thus the evolution of the level-set through (9). Since the B-spline kernel $b_{h}^{n}[\mathbf{u}]$ is separable, the gradient may indeed be computed as a simple series of $d$ convolutions of the feature image with a one-dimensional B-spline kernel, followed by downsampling by $h$. In practice, we use mirror boundary conditions.

\section{IMPLEMENTATION}

\subsection{Bounded Level-Set}

In the course of propagation, the level-set function may develop steep or flat gradients, which, in turn, yield inaccuracies in the numerical approximation [11]. This is usually taken into account in classical implementations by reshaping the level-set through periodical re-initialization of the level-set function as the distance function to the zero level. As noted in [11], such a strategy makes the level topologically less flexible, since it prevents the level-set to develop new contours (i.e., new zero-level components). As shown in [4], bounding the level-set function allows one to avoid this re-initialization step, making the solution topologically more flexible. Such a bounding may be easily performed due to the fact that the level-set function is expressed as a linear combination of basis functions. Using B-spline properties, it can be easily shown that the $\ell_{\infty}$-norm of the B-spline coefficients provides us with a bound on the level-set function, i.e. formally:

$$
|\phi(\mathbf{x})| \leq\|c\|_{\infty} .
$$

Hence, we can normalize the level-set function to the range $[-1,1]$ by the following simple modification of the initial algorithm provided by (9):

$$
\begin{gathered}
\mathbf{c}^{(i+1)}=\mathbf{c}^{(i)}-\lambda \nabla_{\mathrm{c}} J\left(\mathbf{c}^{(i)}\right), \\
\mathbf{c}^{(i+1)} \leftarrow \frac{\mathbf{c}^{(i+1)}}{\left\|\mathbf{c}^{(i+1)}\right\|_{\infty}} .
\end{gathered}
$$

\subsection{Gradient descent algorithm}

The minimization of the energy given in (9) is implemented using a gradient descent with feedback step adjustment. At each step $i$, a candidate update $\mathbf{c}^{\mathbf{i}+\mathbf{1}}$ and the associated energy are computed from the current estimate $c^{i}$ using (9). If this update yields the energy to decrease, the step is considered as successful, the corresponding B-spline coefficients $\mathbf{c}^{\mathbf{i}+\mathbf{1}}$ are accepted and the step size $\lambda$ is multiplied by a factor $\alpha_{f} \geq$ 1. Otherwise, a more conservative update is calculated by dividing the step size by $\alpha_{f}^{\prime} \geq 1$, and the test is repeated.

\section{EXPERIMENTS}

We now validate the proposed approach by considering its application to various types of biomedical images. In all experiments, the images have an eight-bit dynamics and their dimension is $256^{d}$ (where $d$ is the number of dimensions). A cubic B-spline function is used as a basis for the level-set representation. The parameters of the gradient descent step adjustment are fixed as $\alpha_{f}=1$ and $\alpha_{f}^{\prime}=1.5$. In each case, the level-set is initialized using the following implicit function: $\phi_{0}(x, y)=-\sqrt{(x-128)^{2}+(y-128)^{2}}+110$.

We give in Figure 1 an example of segmentation of a fluorescence microscopic image of yeast. The curvature term is applied in this example by setting $\nu=0.01 * 255^{2}$. Figure 1(b) shows the final segmentation, where the multiple components in the image are detected thanks to the topological flexibility of the level-set. The final result is obtained in 6 seconds $c p u$ time.

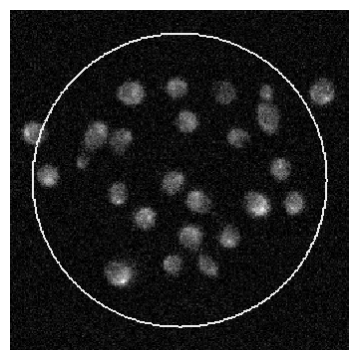

(a) Initialization

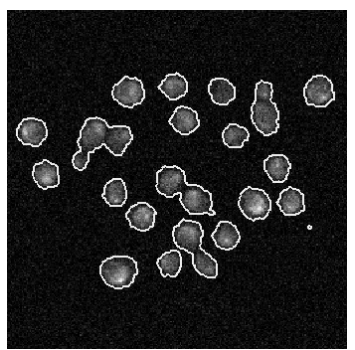

(b) final result, 29 iterations
Fig. 1. Segmentation of a fluorescence microscopic image of yeast with curvature weight $\nu=0.01 * 255^{2}$, cpu $=6 \mathrm{~s}$

We give in Figure 2 an example of application of the approach on a fluorescence microscopic image of a cell. The curvature term is applied in this example by setting $\nu=0.02 *$ $255^{2}$. Figure 2(b) show the final segmentation.

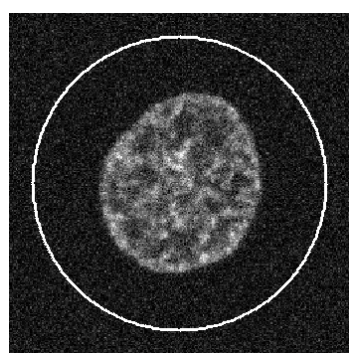

(a) Initialization

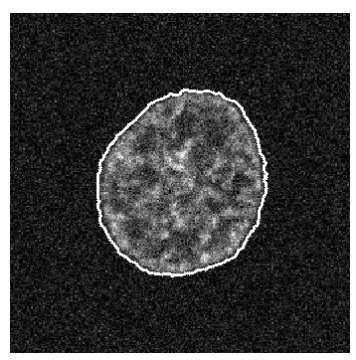

(b) final result, 13 iterations
Fig. 2. Segmentation of a fluorescence microscopic image of cell with curvature weight $\nu=0.02 * 255^{2}$, cpu $=5 \mathrm{~s}$

As shown in Figure 3, we have also applied the proposed segmentation approach to a 3 -D image of a calcaneus bone, acquired on a micro-CT with a voxel size of $80 \mu \mathrm{m}^{3}$. The segmentation was obtained without curvature term to preserve the details structure of the calcaneus bone structure. We provide in Figure 3 a 3-D visualization of the resulting segmentation, as well as two image slices. These results show the ability of the model to handle complex topology. 


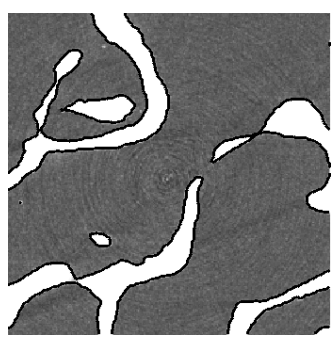

(a)

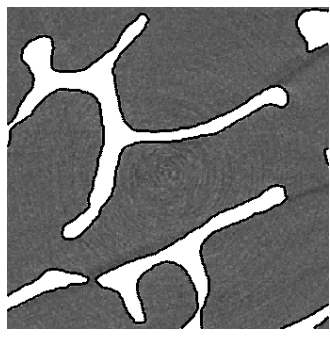

(b)

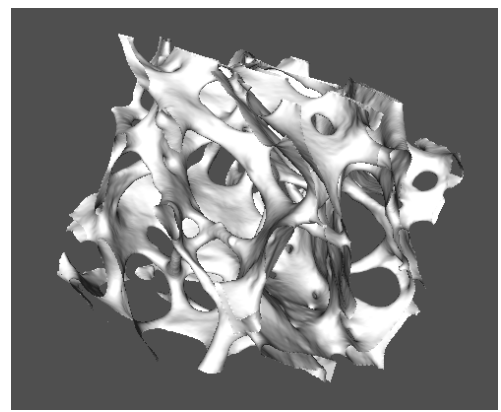

(c)

Fig. 3. Segmentation of 3-D micro-CT images of a calcaneus bone. Level-set model without any curvature term $(\nu=0)$. (a)-(b) Two slices through the original data volume, along with the obtained contours; (c) 3-D rendering of the resulting segmentation. The cpu time is $540 \mathrm{~s}$.

\section{CONCLUSIONS}

We proposed in this paper a new formulation to level-setbased active contours, where the implicit function is modelled as a continuous parametric function expressed on a Bspline basis. This representation provides an overall control of the level-set, and allows avoiding the reinitialization step of the level-set via the normalisation of the B-spline parameters. The B-spline formulation allows to express the level-set evolution as a sequence of $1 \mathrm{D}$ convolution, yielding an efficient algorithm. The behaviour of the proposed approach has been evaluated from biological images from various field. The obtained segmentation results show the interest of the method in terms of computation time and flexibility.

\section{REFERENCES}

[1] J.S. Suri, K. Liu, S. Singh, S.N. Laxminarayan, X. Zeng, and L. Reden, "Shape recovery algorithms using level sets in 2-D/3-D medical imagery: A state-of-the-art review," IEEE Transactions on Information Technology In Biomedicine, vol. 6, no. 1, pp. 8-28, 2002.

[2] S. Osher and R. Fedkiw, Level Set Methods and Dynamic Implicit Surfaces, New York: Springer-Verlag, 2002.

[3] B.S. Morse, W. Liu, T.S. Yoo, and K. Subramanian, "Active contours using a constraint-based implicit representation," in SIGGRAPH '05: ACM SIGGRAPH 2005 Courses, New York, NY, USA, 2005, p. 252, ACM Press.

[4] A. Gelas, O. Bernard, D. Friboulet, and R. Prost, "Compactly supported radial basis functions based collocation method for level-set evolution in image segmentation," IEEE Transactions on Image Processing, vol. 16, pp. 1873-1887, 2007.

[5] T. Chan and L. Vese, "Active contours without edges," IEEE Transactions on Image Processing, vol. 10, pp. 266-277, 2001.

[6] D. Mumford and J. Shah, "Optimal approximation of piecewise smooth functions and associated variational problems," Commun. Pure Appl. Math., vol. 42, pp. 577-685, 1989.

[7] M. Unser, "Splines: A perfect fit for signal and image processing," IEEE Signal Processing Magazine, vol. 16, no. 6, pp. 22-38, November 1999.

[8] G. Aubert, M. Barlaud, O. Faugeras, and S. JehanBesson, "Image segmentation using active contours: Calculus of variations or shape gradients?," SIAM Applied Mathematics, vol. 63, no. 6, pp. 2128-2154, 2003.

[9] H. Zhao, T. Chan, B. Merriman, and S. Osher, "A variational level set approach to multiphase motion," J. Comput. Phys., vol. 127, pp. 179-195, 1996.

[10] M. Unser, A. Aldroubi, and M. Eden, "B-spline signal processing: Part I-Theory," IEEE Transactions on Signal Processing, vol. 41, no. 2, pp. 821-833, February 1993.

[11] R. Tsai and S. Osher, "Level set methods and their applications in image science," Communications in Mathematical Sciences, vol. 1, no. 4, pp. 1-20, 2003. 